\title{
Three-Dimensional (3D) Ultrasound and Doppler Angiography Imaging in the Prenatal Assessment on Normal and Abnormal Fetal Cardiac Anatomy
}

\author{
Wael El Guindi ${ }^{1 *}$, Mohamed Youssef ${ }^{2}$, Gabriel Carles ${ }^{1}$ Michel Dreyfus $^{3}$ and Sameh Elsayed \\ ${ }^{1}$ Department of Obstetrics and Gynecology, Franck Joly Hospital, Saint-Laurent-du-Maroni, France \\ ${ }^{2}$ Department of Obstetrics and Gynecology, Polyclinique de Blois, Blois, France \\ ${ }^{3}$ Department of Obstetrics and Gynecology, Caen University Hospital, Caen, France \\ ${ }^{4}$ Department of Obstetrics and Gynecology, Cairo University, Egypt
}

*Corresponding author: Wael El Guindi, Department of Obstetrics and Gynecology, Franck Joly Hospital, Saint-Laurent-du-Maroni, France

Received: October 21, 2021; Accepted: November 03, 2021; Published: January 03, 2022

\begin{abstract}
Objective: To highlight the value of 3D volume ultrasound in the prenatal assessment on normal and abnormal fetal cardiac anatomy.

Methods: A retrospective offline analysis of volume datasets of 160 pregnant women carrying fetuses with normal cardiac anatomy and 40 pregnant carrying fetuses with cardiac malformations was conducted.

Results: 45 cardiac malformations were diagnosed. Isolated cardiovascular malformations were detected in 27 fetuses, of which 4 had trisomy 21 and 1 had trisomy 18 one fetus exhibited 22q11 microdeletion (DiGeorge syndrome). Extracardiac abnormalities were identified in 13 fetuses.

Conclusion: 3D ultrasound offers a high-resolution volume rendering image that provides excellent delineation of fetal cardiac anatomy and add significantly to understanding of the normal and abnormal cardiovascular anatomy, an axiomatic element, to understand cardiovascular anomalies, moreover, these high-quality images clarifying fetal cardiac anatomy may be effective in teaching basic fetal echocardiography.
\end{abstract}

Keywords: Fetal echocardiography, Power doppler imaging, Prenatal diagnosis, 3D ultrasound, 4D ultrasound, Cardiovascular malformations, Ultrasound, Congenital Heart Disease (CHD)

\section{Introduction}

Congenital Heart Disease (CHD) are structural abnormalities of the heart or intra-thoracic great vessels arising before birth $[1,2]$. They are the most common congenital malformations and the most frequently overlooked during prenatal ultrasonographic scanning with an estimated prevalence of 8 in 1000 live births $[3,4]$.

It is an axiomatic fact that recognition of abnormality from fetal heart require an in-depth understanding of the normal fetal cardiovascular anatomy. This knowledge is necessary to fully understand different types of congenital heart defects. Echocardiography remains the preferred modality for assessment of the fetal heart for congenital heart disease [5].

We conducted this study on 160 pregnant women carrying fetuses with normal cardiac anatomy and 40 pregnant women carrying fetuses with cardiac malformations. Examinations were performed via Voluson 730 Pro (General Electric, Milwaukee, WI, USA) with a volumetric abdominal transducer (4-8 MHz). The examination, rendering and display of stored cardiovascular volumes datasets were performed by one independent examiner who was not blinded to the previous diagnoses of cardiac anomalies per 2D ultrasound.

Sonography from patients with suspected cardiac anomalies was then sent via the internet to a prenatal diagnosis reference center (Caen Teaching Hospital, France), and the initial diagnosis was confirmed or revised. A multidisciplinary team including a pediatric cardiologist, a neonatologist, and a pediatric cardiac surgeon provided comprehensive prenatal counseling to each expectant mother. Neonatal echocardiography was used to confirm the prenatal diagnosis in surviving fetuses. Autopsy findings from terminated pregnancies were compared to fetal echocardiograms.

\section{Results}

In total, 160 pregnant women carrying fetuses with normal cardiac anatomy and 40 pregnant women carrying fetuses with 45 cardiac malformations were evaluated (Table 1). Mean gestational age at diagnosis was 26 weeks (range, 20-34 weeks). In the second group, isolated cardiovascular malformations were detected in 27 fetuses, extracardiac abnormalities were identified in 13 fetuses, 4 fetuses had trisomy 21 and 1 had trisomy 18 . One fetus exhibited $22 \mathrm{q} 11$ microdeletion (DiGeorge syndrome). The 2D ultrasound diagnosis was revised after offline analysis of cardiovascular volumes for 4 cases: transposition of the great arteries was revised to double outlet right ventricle; in which atrioventricular canal defect was revised to persistent left superior vena cava interventricular septal defect was revised to atrioventricular canal defect; cardiomegaly was revised to 
Table 1: Cardiac malformations diagnosed.

\begin{tabular}{|c|c|c|c|c|}
\hline Cardiac anomaly & No. & Associated cardiac anomalies & Extracardiac anomalies & Chromosomal anomalies \\
\hline Situs inversus & 3 & - & - & - \\
\hline Situs ambiguus or heterotaxy & 10 & $\begin{array}{c}10 \text { (7 cases of interruption of IVC with } \\
\text { azygos continuation) }\end{array}$ & 7 & - \\
\hline Dextrocardia & 2 & - & 1 & - \\
\hline Tetralogy of Fallot & 1 & - & 1 & - \\
\hline Transposition of the great arteries & 2 & - & - & - \\
\hline Pulmonary valve stenosis/pulmonary valve atresia & 2 & 2 & - & DiGeorge syndrome $(\mathrm{n}=1)$ \\
\hline Single ventricle & 4 & 3 & - & - \\
\hline Aortic coarctation & 2 & - & - & - \\
\hline Atrioventricular canal defect & 5 & 3 & 2 & Trisomy $21(\mathrm{n}=3)$ \\
\hline Persistent left superior vena cava & 3 & 1 & 1 & - \\
\hline Aneurysm of foramen ovale & 2 & - & - & - \\
\hline Double outlet right ventricle & 2 & - & - & - \\
\hline Hypoplastic aortic arch & 1 & 1 & 1 & Trisomy $18(\mathrm{n}=1)$ \\
\hline Extrahepatic umbilical vein & 2 & - & 1 & - \\
\hline Abnormal course of left hepatic vein & 2 & - & 2 & - \\
\hline $\begin{array}{l}\text { Right aortic arch } \\
\text { Total anomalous pulmonary venous return (TAPVR) }\end{array}$ & $\begin{array}{l}1 \\
1\end{array}$ & - & - & - \\
\hline Total & 45 & 20 & 16 & 4 \\
\hline
\end{tabular}

extrahepatic umbilical vein (6L). A misdiagnosis of aortic coarctation was made via 3D ultrasonography in 1 case; however, this was rectified after reanalysis of cardiac volume.

Grayscale largely facilitated the diagnosis of situs (Figures 1-5). Power Doppler imaging contributed primarily to prenatal diagnosis of vascular anomalies, and it was extremely beneficial in detecting situs (Figure 6A, 6B and 6E). Dextrocardia was diagnosed by Doppler imaging following localization of the apex of the heart and axis of the left hepatic vein on opposite sides (Figure 6E and 6F). By moving through an acquired volumetric datasets of fetus's normal hearts and with fetuses with Congenital Heart Disease (CHD), we have been able to display normal and abnormal fetal cardiac anatomy, to obtain realistic anatomic image with no mental reconstruction of spatial relationships thus enhancing our understanding of anatomic relationships. Despite the use of an ultrasound machine that does not have STIC technology, we have been able to display images, especially fetal cardiac valves, to our knowledge, not yet obtained with devices having recent technology like STIC, FINE, highlighting the inherent capacity of 3DUS which has not been fully exploited. In our previous work, we have shown the added value of 3D volume in delineation of the gastrointestinal tract aberrant anatomy of intestinal malrotation caused by Ladd's bands [6].

\section{Discussion}

$3 \mathrm{D}$ volume is like clay, can be moulded into different shapes, the only difference is that the $3 \mathrm{D}$ volume can be shaped into predetermined shapes, i.e. anatomy; it is up to the operator to retrieve anatomic components of the fetal heart from these volume datasets. To better understand this concept, we refer the reader to our work in which D-TGV was diagnosed by off-line analysis from only one stored 3D volume of 4-chamber view [7].

$3 \mathrm{D}$ volume datasets are static by nature as they only acquire spatial information which contains an infinite number of adjacent $2 \mathrm{D}$ with no regard to temporal or spatial motion $[8,9]$.

For example, in the 4-chamber view the anatomy of the heart and great vessels at the moment of acquisition i.e., according to the phase of the cardiac cycle, is contained and stored in volume datasets, the main value of $3 \mathrm{D}$ volume is the ability unveil the anatomical information contained i.e., reconstruct 3D spatial images standard planes on a $2 \mathrm{D}$ screen in a process called rendering [10]. To obtain volume database during a complete cardiac cycle, temporal dimension information that is present during acquisition has to be incorporated, Spatio-Temporal Image Correlation (STIC) is a new a software modality that unites the spatial and temporal domains resulting in a volume of a complete fetal cardiac cycle displayed in motion by means of a sequence of $3 \mathrm{D}$ cineloop that consists of a high number of $2 \mathrm{D}$ frames, one behind the other $[11,12]$. However, extracting and displaying meaningful information from volume datasets is highly expertise-dependent, difficult to perform and require a sound knowledge of fetal anatomy [12]. It has recently been shown that applying "intelligent navigation" technology to STIC the FINE method can automatically generates and displays eight to nine standard fetal echocardiography views [13]. For a more in-depth description of the various techniques, readers are directed to the original references. The availability of several display modes and standardized examinations permits the demonstration of both the normal and abnormal fetal anatomy in controlled planes and rendered images from different angles [14]. 
Wael El Guindi (2021) Three-Dimensional (3D) Ultrasound and Doppler Angiography Imaging in the Prenatal Assessment on Normal and Abnormal Fetal Cardiac Anatomy

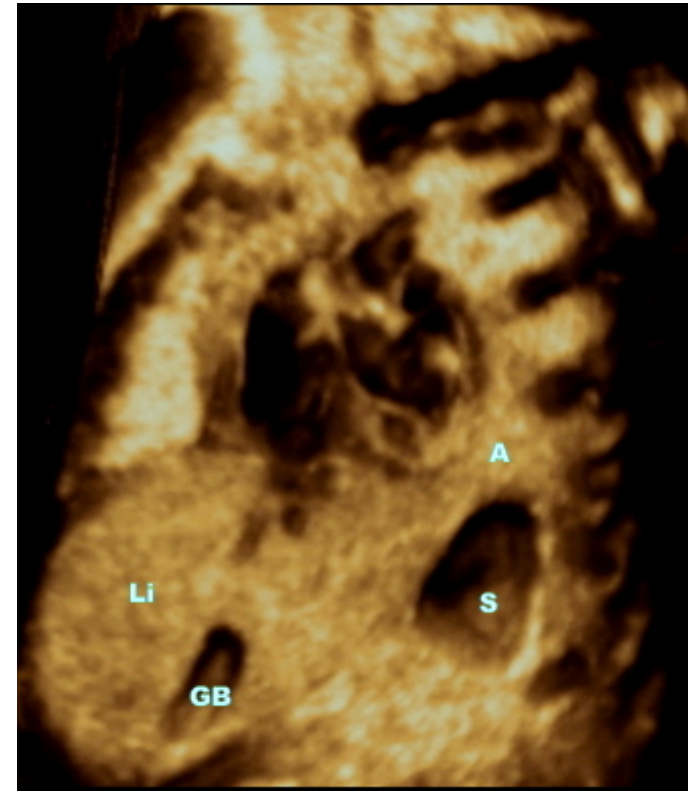

A

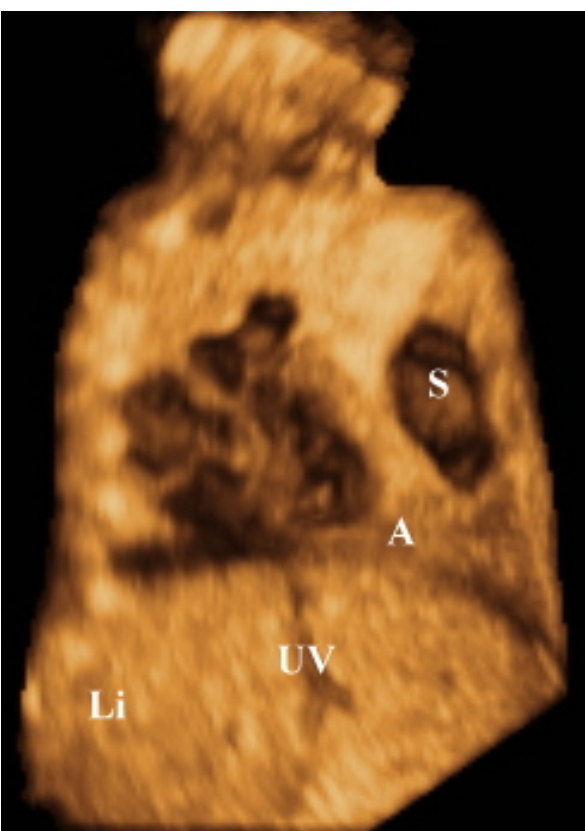

D

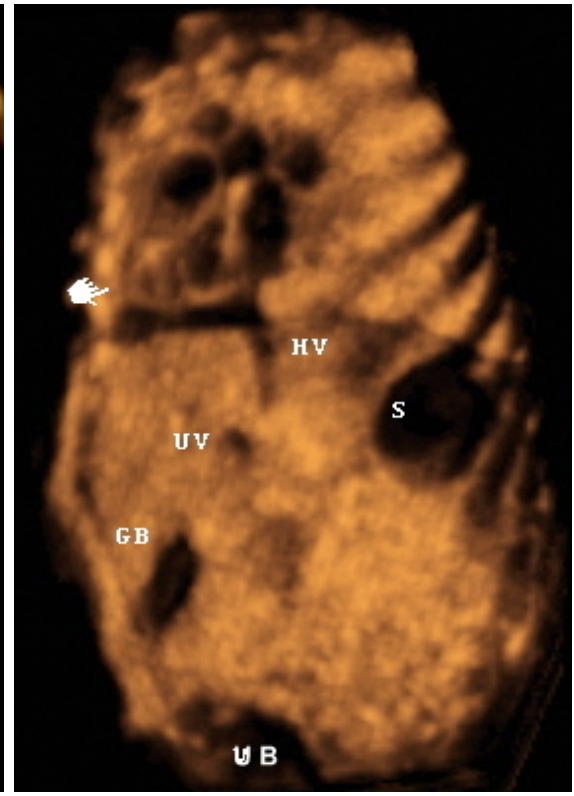

B

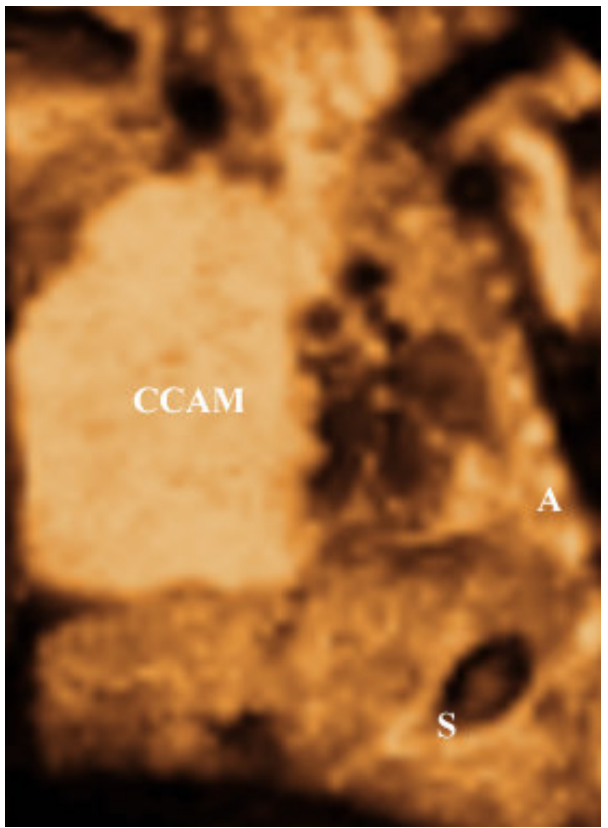

E

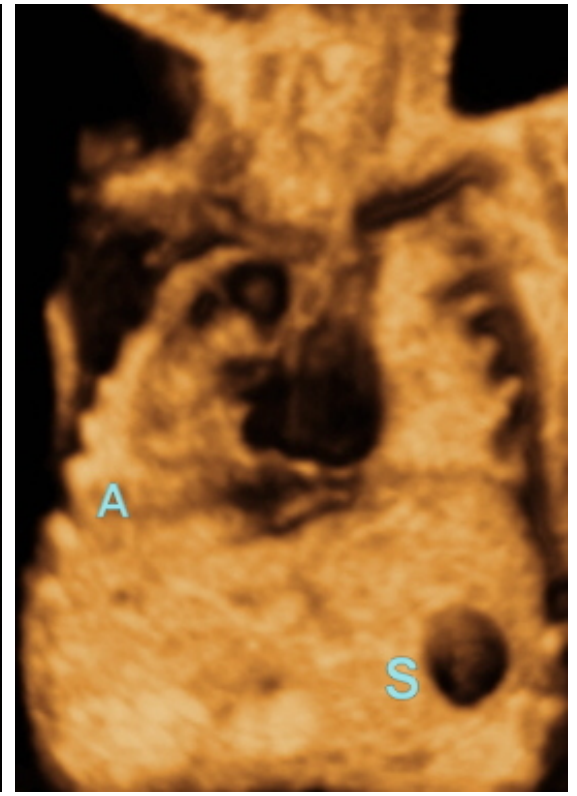

C

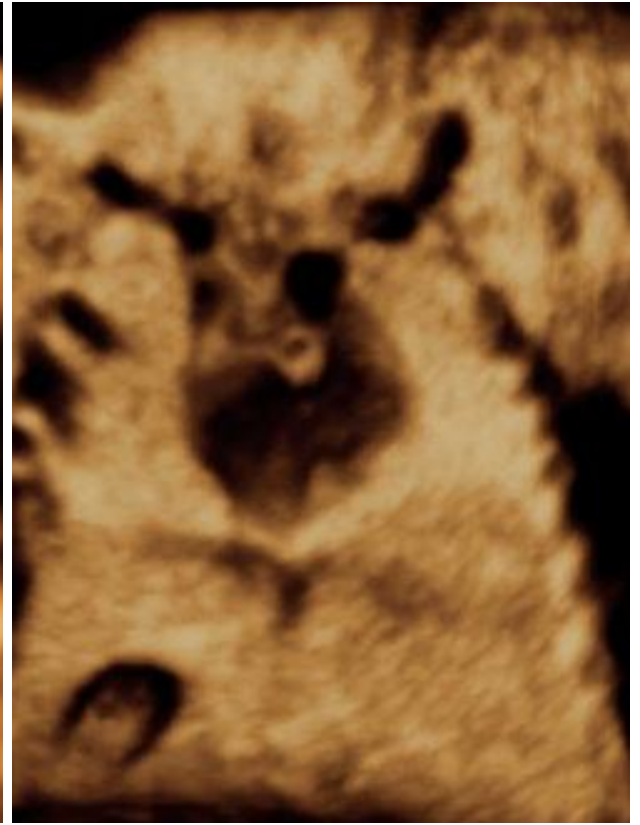

F

Figure 1: Grey-scale volume-rendered images. Situs determination.

(A) Levocardia. the heart is mainly situated in the left hemithorax, with the apex pointing to the left and stomach are on the left; the gallbladder is on the right.

(B) Dextrocardia, heart in the right chest, apex pointing rightward.

(C) Dextrorotation: the base of the heart is in the normal position, but the apex has rotated rightwards

(D) Dextroposition, rightward displacement of the heart with apex (A) pointing leftward, in (left-sided diaphragmatic hernia).

(E) Levoposition: The heart is mainly located in the left chest due to (CCAM) Congenital Cystic Adenomatoid Malformation - confirmed by fetopathological examination; cardiac apex is to the left (same side as stomach).

(F) Mesocardia, the heart is positioned in the middle of the chest, with the apex pointing to the midline.

Abbreviations: A: apex of the heart Stomach; VB: gallbladder; VCI: inferior vena cava. CCAM: Congenital cystic adenomatoid malformation. UV: umbilical vein. H: heart. UB: urinary bladder. Li: liver. 
Wael El Guindi (2021) Three-Dimensional (3D) Ultrasound and Doppler Angiography Imaging in the Prenatal Assessment on Normal and Abnormal Fetal Cardiac Anatomy

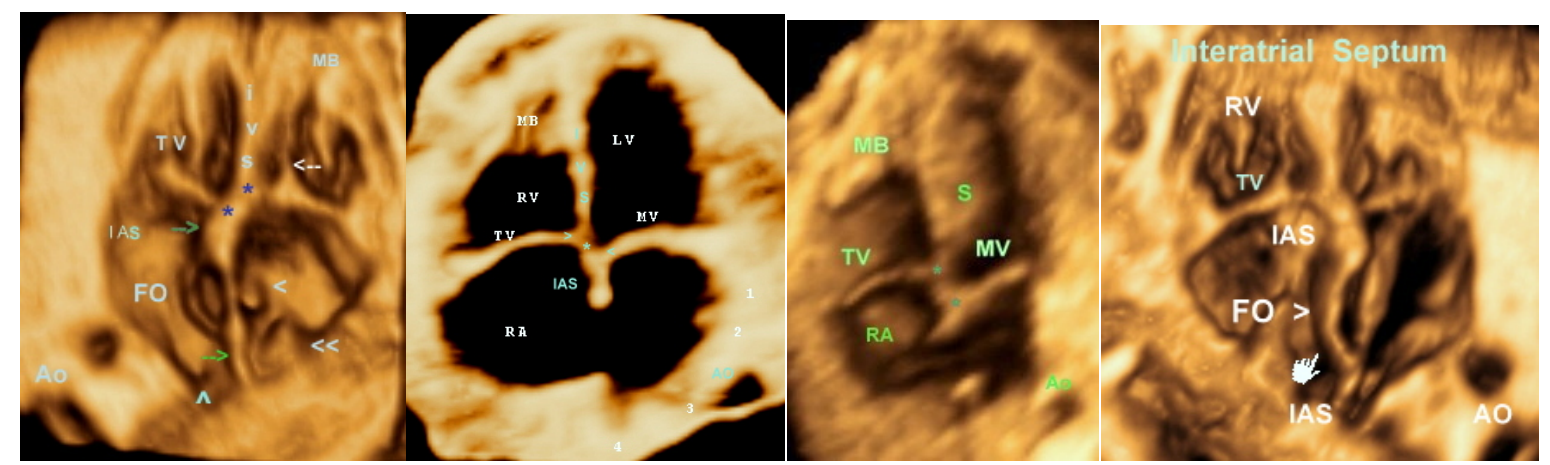

A

B

C

D

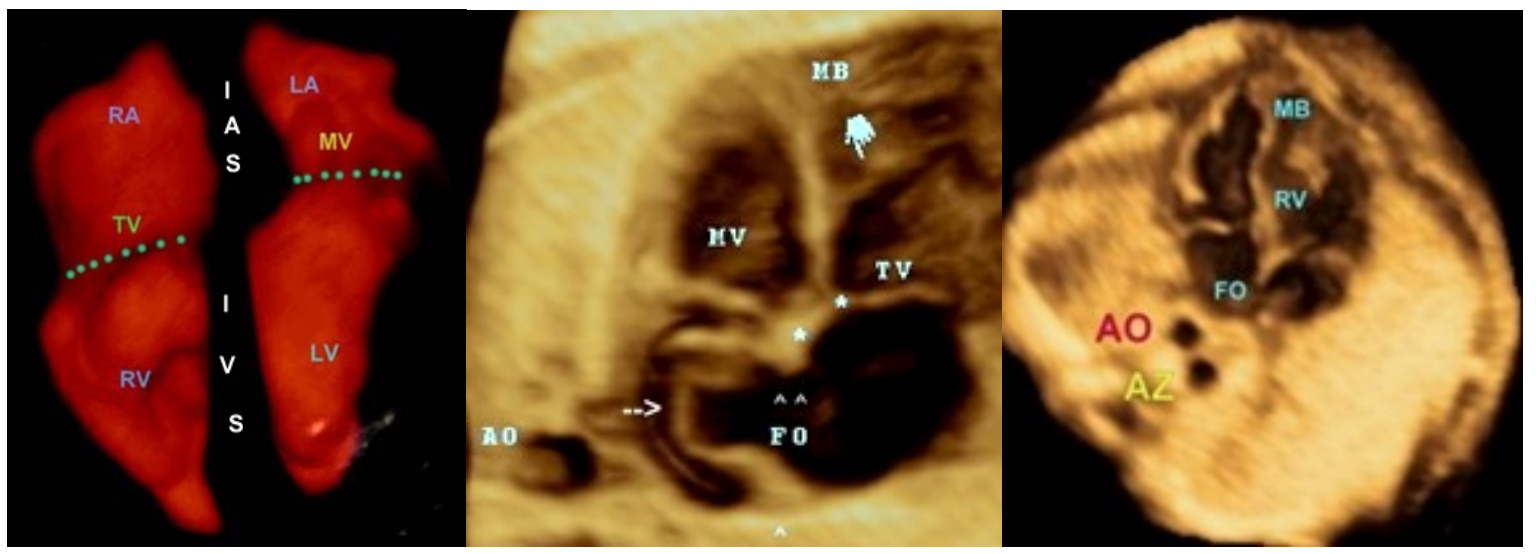

E

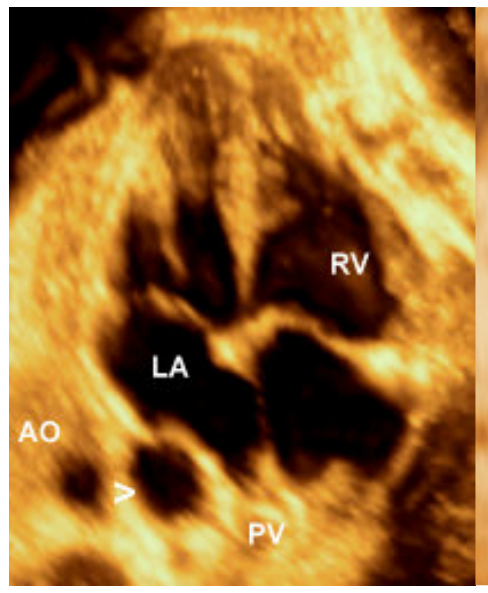

H
F

G

Figure 2: Normal and abnormal Four-chamber view.

A,B,C,D and E: Four-chamber view in a normal fetus showing

- Foramen ovale pointing to the left.

- Apical displacement of the tricuspid valve septal leaflet insertion when compared with septal insertion of the mitral valve(asterisks)

- Septal leaflet of the Tricuspid valve. (White arrow).

- Interventricular Septum (IVS).

- Interatrial Septum (IAS).

$>$ SVC $>>$ IVC Green arrows IAS

(F) Atrial septal aneurysm (Aneurysm of the foramen ovale).

(G) A dilated azygos vein (Az) posterior to the descending aorta (Ao) "Double vessel sign."

(H) Distended esophagus (arrowhead). Esophageal duplication cyst that can be confound with a dilated Azygos vein, color doppler easily differentiate between them.

(I, J) Ebstein's anomaly. Note the apical displacement of the tricuspid valve and "atrialization" of the base of the RV. > denotes septal leaflet of the TV ,>> denotes anteropr leaflet of the TV. 


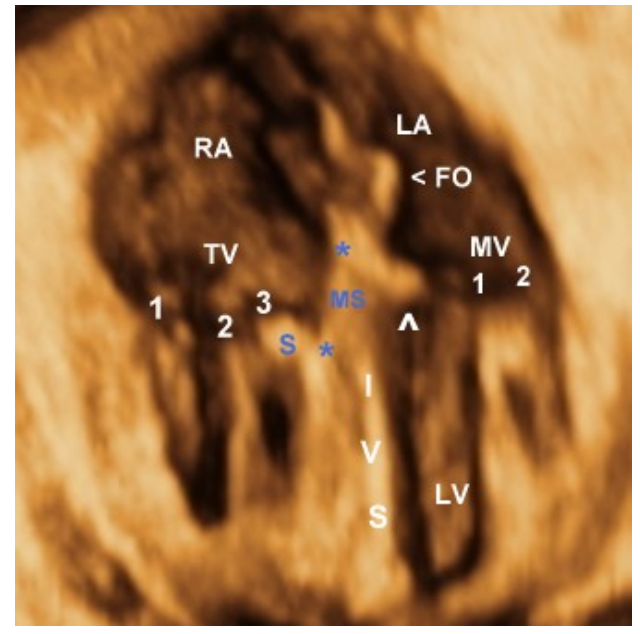

A

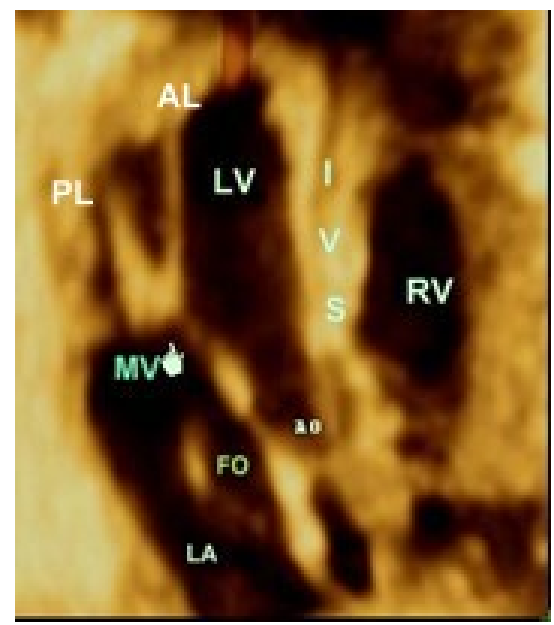

$\mathrm{E}$

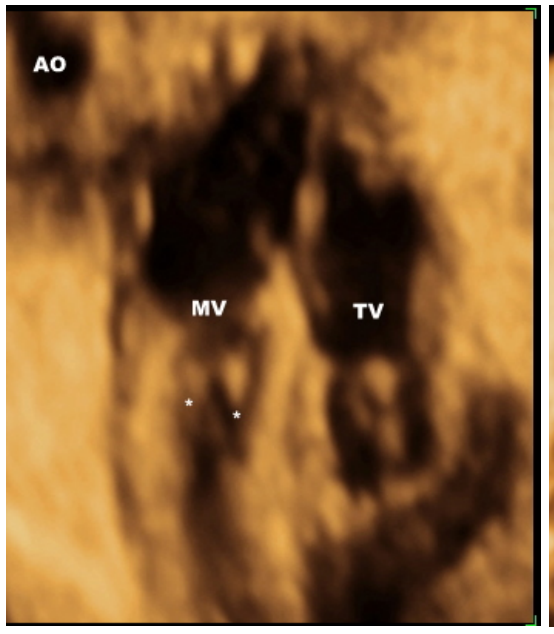

B

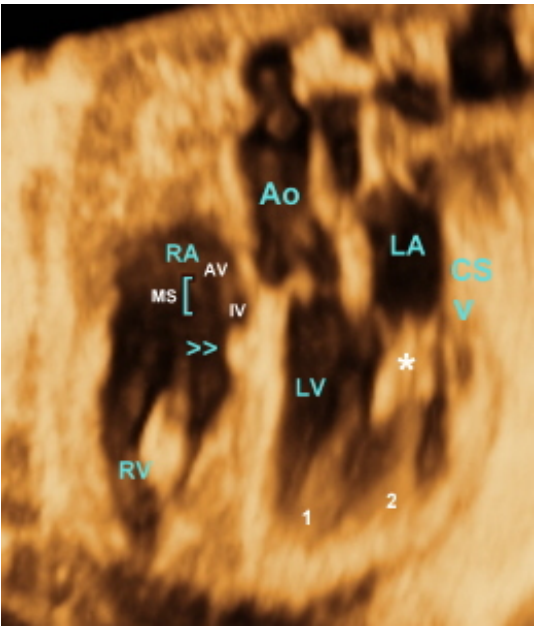

$\mathrm{C}$

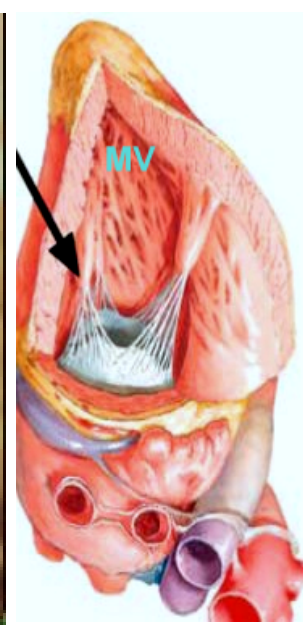

$\mathrm{F}$

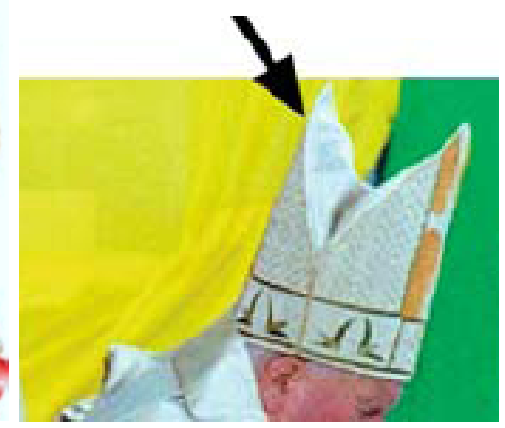

G

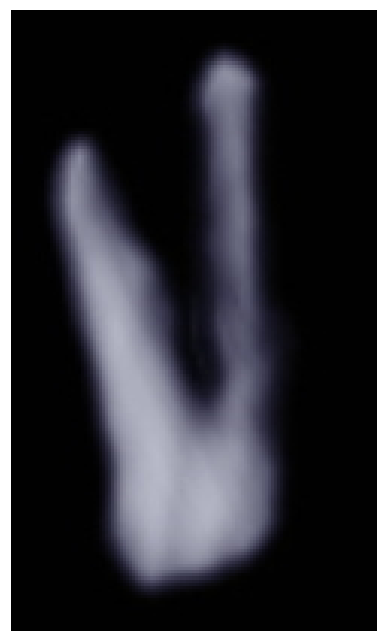

$\mathrm{H}$

Figure 3: Atrioventricular valves.

A,B-Volume-rendered image showing the Tricuspid Valve (TV) that consists of 3 leaflets, with the characteriatic septal leaflet (S), the mitral valve has 2 leaflets with no attachments to the septum. C-Membranous part of the IVS, papillary mucle(PM) and the chordae tendineae ${ }^{*}$ ) of the mitral valve

D, E,F- Mitral valve, the MV (left atrioventricular or bicuspid) is so named because of its resemblance to a cardinal's hat, known as a mitre.

$\mathrm{H}$ - Volume-rendered image showing the mitral the leaflets valve (MV) Note that anterior leaflet is longer than the posterior leaflet. 1, antero-lateral papillary muscle; 2,PMPM, postero-medial papillary muscle.IA, interatrial septum ,IV, interventricular septum.

Three- and four-dimensional (3D/4D) ultrasound in offers several distinct advantages to fetal echocardiography [15]. To assess cardiac situs, it is essential to determine which the right is and left side of the fetus [16], to determine fetal visceral situs, fetal head and spine must be checked and compared with maternal spine [17]. However, this approach can be inherently difficult and affected by fetal position or movements; in addition, this method is not reliable if there is a malposition of an indicator organ [18].

Our current experience shows that grayscale offer great advantages in the diagnosis of situs (Figure 1-3). 3D rendered images clearly showed the position of heart in the thorax, the direction apex of the heart with regard to stomach without the need to reconstruct the spatial relations between the fetus and the mother. Power Doppler imaging contributed primarily to prenatal diagnosis of vascular anomalies, and it was extremely beneficial in detecting situs (Figure 4A and 4B).

Because the definition of dextrocardia has generated a lot of controversy [19] in this article we adopt the definition of dextrocardia as the heart being in the right hemithorax, and the apex is oriented to the right (Figure 1 B) $[20,21]$. Dextroposition, the heart is shifted into right hemithorax the right chest as a result of extracardiac abnormalities, with cardiac apex pointing to the left while (Figure 1D) [22]. Dextrorotation (Figure 1C), the base of the heart is in the normal position, but the cardiac apex points to the right [23]. Mesocardia, the heart is positioned in the middle of the chest; the apex is in a midline position (Figure 1F) [21]. Dextrocardia was diagnosed by doppler imaging following localization of the apex of the heart and axis of the left hepatic vein (Figure 4B). 


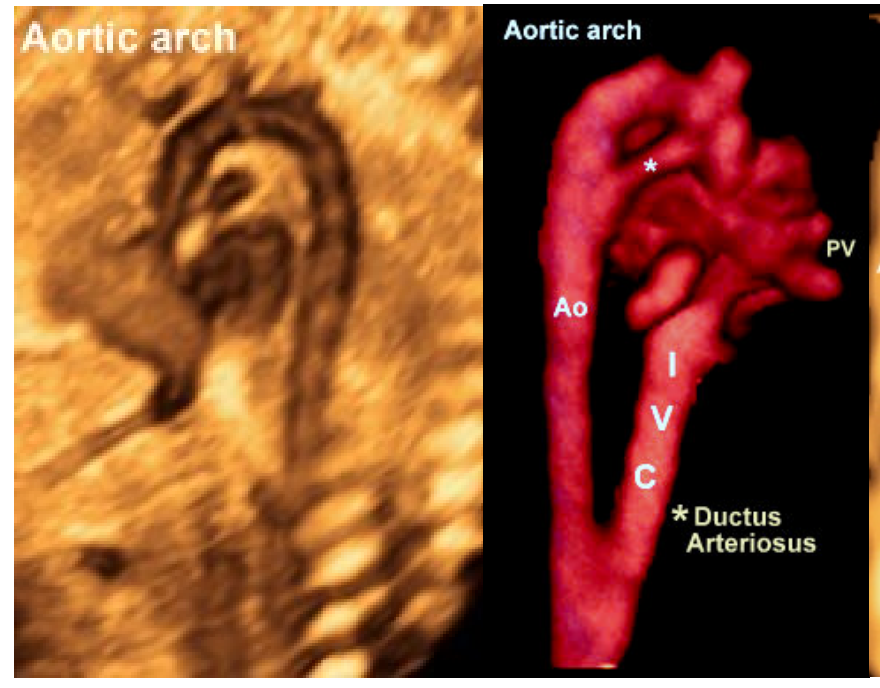

A

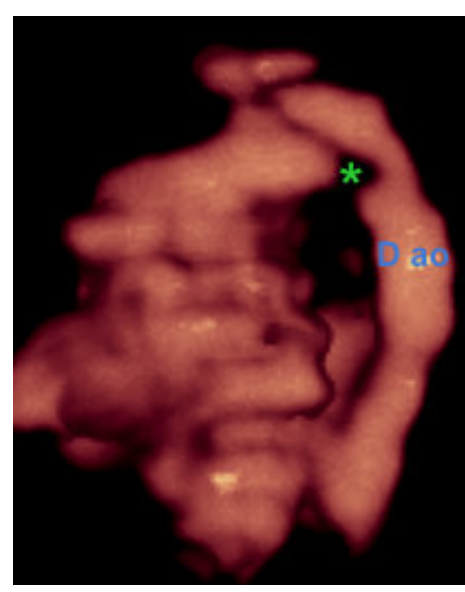

E
B

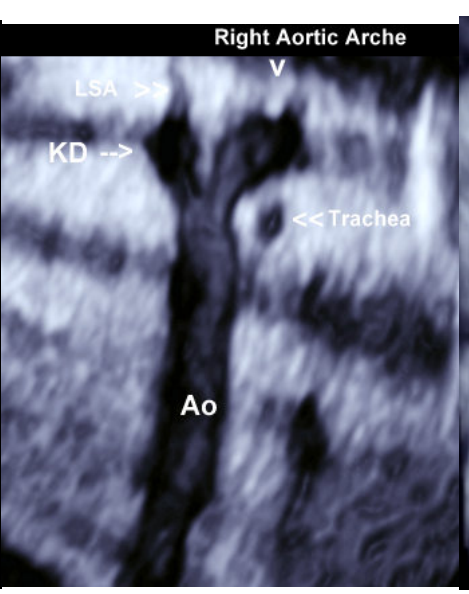

$\mathrm{F}$

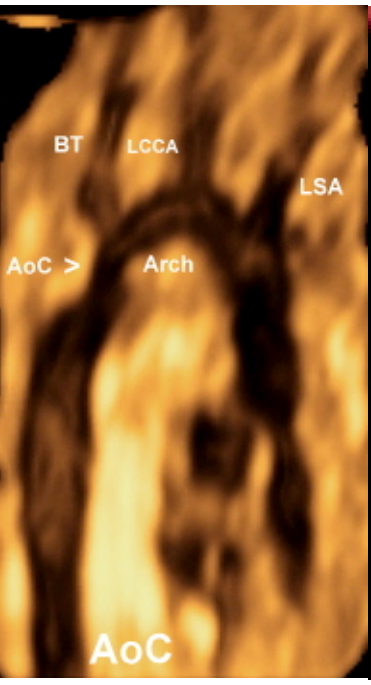

$\mathrm{C}$

D

Figure 4: Normal and abnormal aortic arche.

$(\mathrm{A}, \mathrm{B})$ Normal aortic arch.Asterisk denotes arterial canal.

(C,D,E) Aortic coarctation, note the presence of coarctation shelf (Asterisk).

(F) Right aortic arch with aberrant left subclavian artery arising from a diverticulum of Kommerell.

(G) Right aortic arch, Trachea (T) is located between paralleled pulmonary artery and aorta.

(H) Sagittal image demonstrating the dilated azygos vein and azygos arch connecting to the superior vena cava $\left(^{\star}\right)$. Care should be taken not to confound it with aortic arch.

Abbreviations: LCCA, left common carotid artery; LSA, left subclavian artery;; left brachiocephalic trunk (BT).

KD; Kommerell diverticulum Pulmonary artery AO; Aorta HV; Hepatic vein DV; Ductus venous

EV; Eustachian Valve VC; superior vena cava. Dao; Descending aorta0

From our experience [21-23], both Left Hepatic Vein (LHV) and the apex of the heart are in the same side and point to the same direction i.e., downwards, the cardiac apex points to an opposite direction with respect to the spine i.e., away from the spine (Figure 6A-6E) [20].

Any deviation from this relationship, using either Glass body mode or Doppler angiography would necessities further evaluation, for example, (Figure 6D and 6E) the cardiac apex and the LHV are in opposite directions and the cardiac apex points toward the spine, denoting an abnormal cardiac axis (Dextrocardia). Fetal parasagittal view (Figure $A, B, C, D$ ) showing this relation is easy to obtain and interpret, offer a realistic anatomic image, needs no mental reconstruction of spatial relationships and is very beneficial mainly in detecting the situs and offers a novel technique for the diagnosis of normal and abnormal fetal situs and an added tool for the diagnosis of other anomalies and we recommended to obtain this view, if not routinely, in all cases suspected of situs anomalies using either doppler angiography with or without glass body mode.

With Grayscale we have been able to interactively unveil and display the anatomy fetal cardiac valves. Figure 3 illustrate mitral valve anatomy (left atrioventricular or bicuspid) so named because of its resemblance to a cardinal's hat, known as a mitre. Photo of the Pope that appears on the Vatican Web site [24]. 


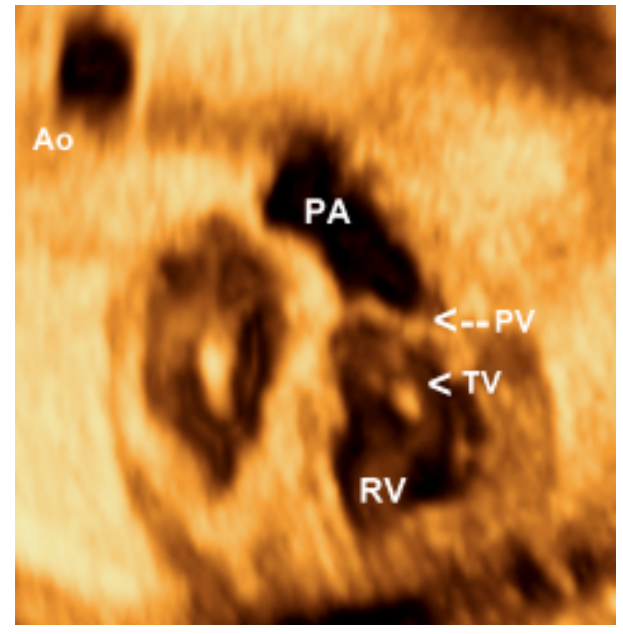

A

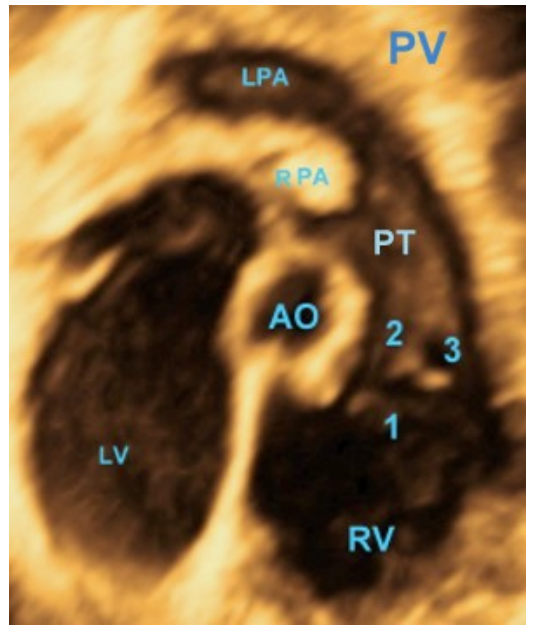

B

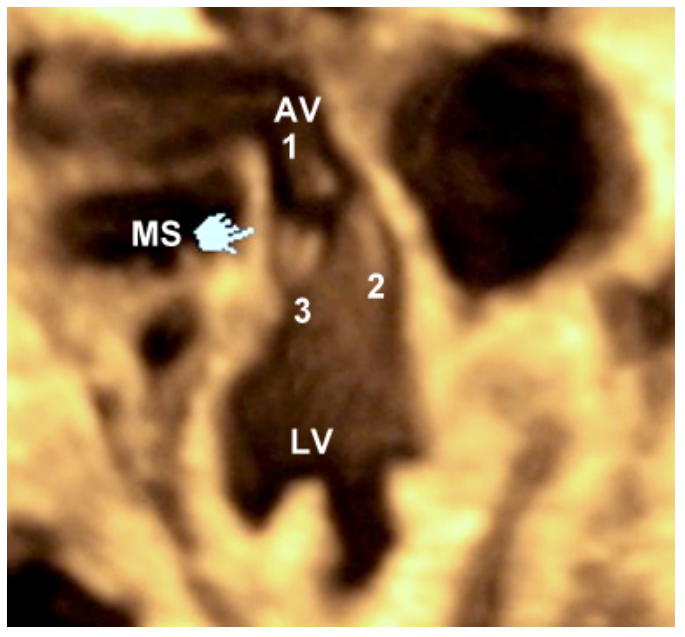

C

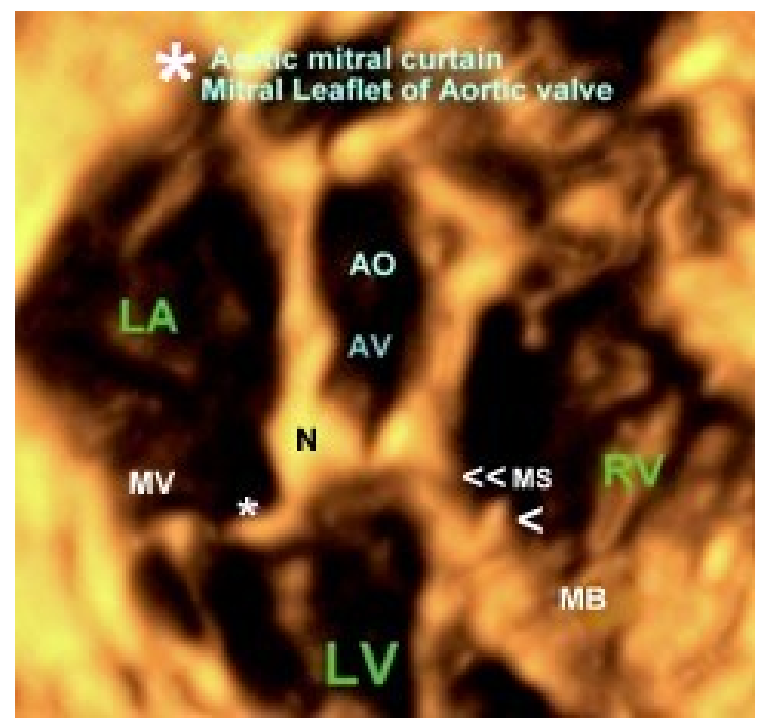

D

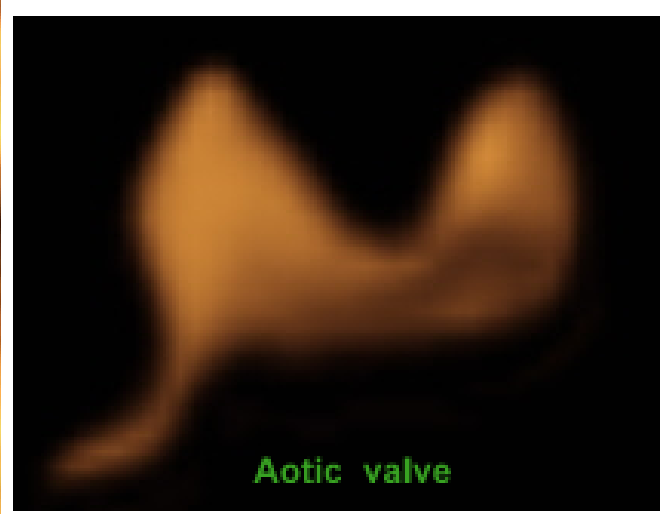

E

Figure 5: Aortic and pulmonary valves.

(A,B) Right Ventricular Outflow Tract (RVOT) showing Trisuspid Valve (TV) and pulmonary valve).

(C) Left Ventricular Outflow Tract (LVOT) showing aortic valve (3 cuspids),

(D,E) Aortic valve , Astricks* denotes mitral leaflet of the aortic valve,arrowhead denotes septal leaflet of the tricuspid valve.

$\gg$ denotes the membranous septum

$\mathrm{N}$ : denotes the noncoronary cusp of the aortic valve.

Astricks*: Mitral leaflet of the aortic valve

Ao: aorta; MPA: Main Pulmonary Artery; PV: Pulmonary Valve; RA: Right Atrium; RPA: Right Pulmonary Artery; RV: Right Ventricle; TV: Tricuspid Ventricle.

The anatomy of the tricuspid valve (Figure 3) with its apical displacement, septal leaflet in both normal and abnormal (Ebstein's) hearts. The three cusps of both pulmonary and aortic valves (Figure 5) are displayed with mitral leaflet of the $\mathrm{AV}$ valve of clearly demonstrated. Moreover, with 3D Power Doppler we have been able to demonstrate A Persistent Left Superior Vena Cava (PLSVC) draining through a dilated coronary sinus into the right atrium with presence of right SVC (Figure 6F and 6I).
With both volumes rendered datasets and doppler angiography we obtained a high-quality image of "contraductal shelf" in a case the aortic coarctation (Figure 4C-4E). In addition, in Figure 6G, fourchamber view showed coronary cameral fistula involving the right coronary artery and right ventricle in a case of pulmonary atresia with an intact ventricular septum (PA-IVS), to our knowledge, this is the first article to illustrate cameral fistula using 3D Power Doppler. 


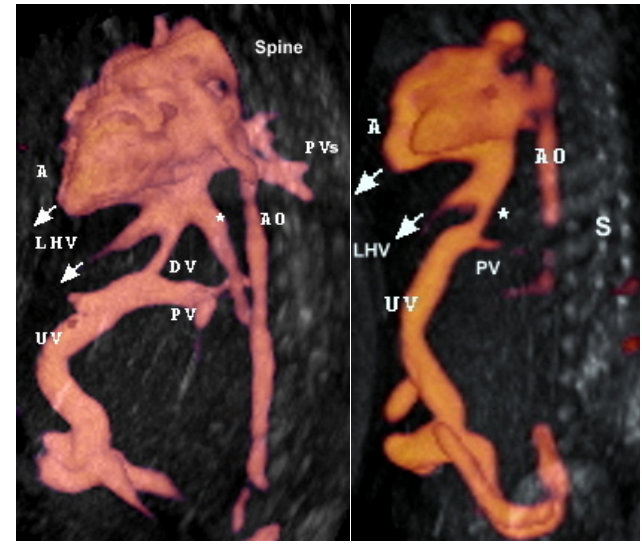

A

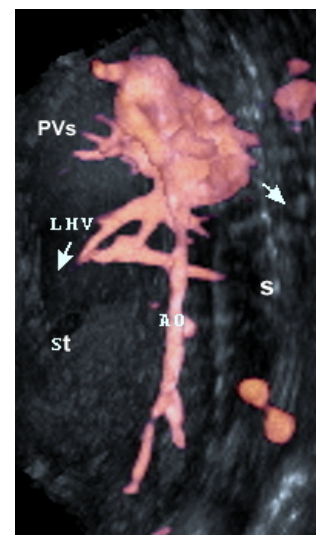

E

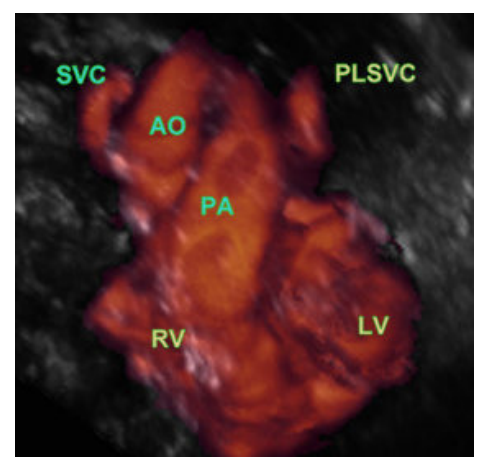

I

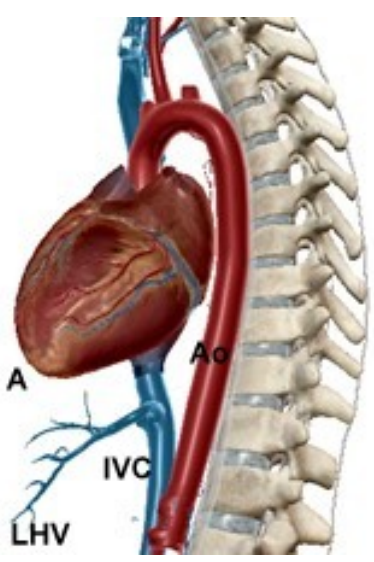

C

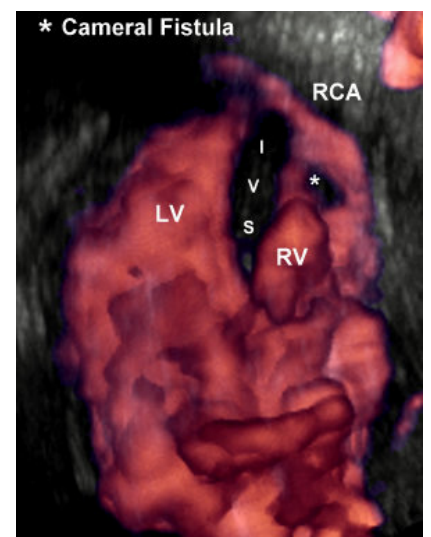

G

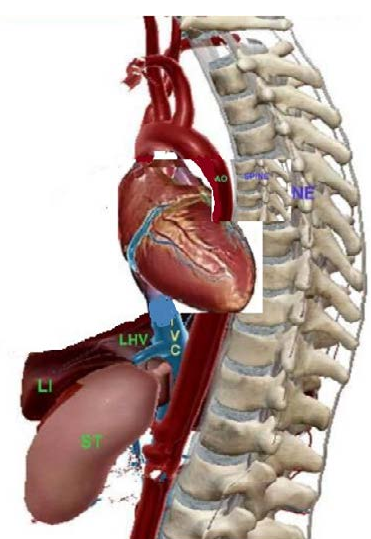

D

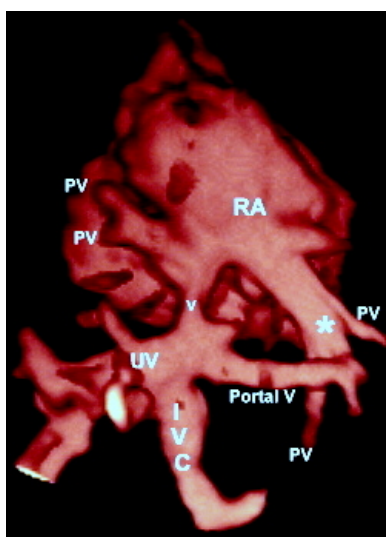

$\mathrm{H}$

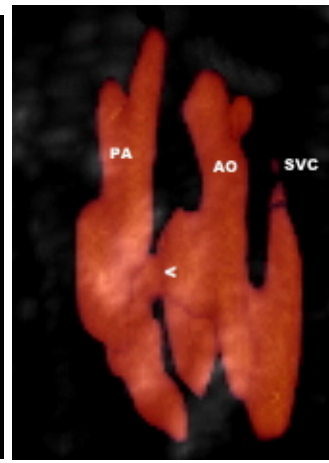

K

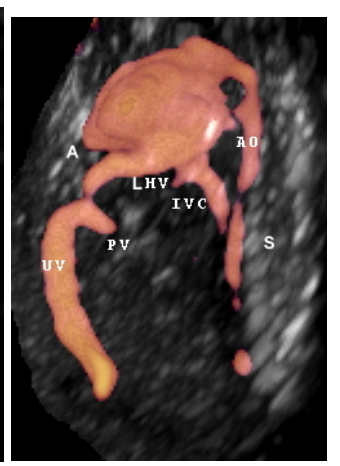

L

Figure 6: Glass body rendred volume.

A. Parasagittal view in a normal fetus showing abdominal vessels of the heart, the apex of the heart, and the left hepatic vein are on the same side, stomach (St) is on left side.

B. Parasagittal view in a fetus with left isomerism and interrupted inferior vena cava. The inferior vena cava is not identified (location marked by the "*” sign).

C. A diagram showing abdominal vessels of the heart, the apex of the heart, and the left hepatic vein are on the same side, stomach (St) is on left side.

D. The heart is turned $180 \mathrm{c}$, i.e. dextrocardia, note that the apex of the heart, and the LHV are on opposite sides (dextrocardia), stomach (St) is on left side. (This is not a real anatomic photo). E. 3D power Doppler ultrasound showing dextrocardia, the apex of the heart, and the spine are on opposite sides, cardiac apex is toward the spine, cardiac apex and LHV are on opposite side.

F. Persistent left superior vena cava draining into the dilated coronary sinus and to the right atrium, the coronary sinus is aneurysmal

G. Pulmonary atresia with an intact ventricular septum (PA-IVS), Asterisk denotes cameral fistula , abnormal communication between right coronary artery and right ventricle.

H. Doppler angiography showing total anomalous pulmonary venous connection with two left and two right pulmonary veins draining into a common vertical vein (V) which drains into infradiaphragmatic inferior vena cava.

I. Normal crisscrossing of the great vessels a Left Persistent Superior Vena Caves (LPSVC).

J. Normal crisscrossing of the great vessels with small pulmonary artery diameter, a case of Tetralogy of Fallot (TOF).

K. Transposition of great arteries, arrow head denotes a ventricular septal defect

L. Extrahepatic umbilical vein draining directly into LA. 
Despite the use of an ultrasound machine that does not have STIC technology, we have been able to display images, especially fetal cardiac valves, to our knowledge, not yet obtained with devices having a more recent technology like STIC, FINE, highlighting the inherent capacity of 3DUS which, in our opinion, has not been fully exploited. By moving through an acquired volumetric dataset of fetuses with normal hearts and with fetuses with Congenital Heart Disease (CHD), we have been able to display normal and abnormal fetal cardiac anatomy, to obtain realistic anatomic image with no mental reconstruction of spatial relationships thus enhancing our understanding of anatomic relationships.

\section{Conclusion}

$3 \mathrm{D}$ ultrasound offers a high-resolution volume rendering image that provides excellent delineation of fetal cardiac anatomy and add significantly to understanding of the normal and abnormal cardiovascular anatomy, an axiomatic element, to understand cardiovascular anomalies, moreover, these high-quality images clarifying fetal cardiac anatomy may be effective in in-depth understanding and teaching basic echocardiography. Offline analysis of cardiovascular anomalies conferred significant diagnostic advantages over standard $2 \mathrm{D}$ and represents an invaluable tool for the prenatal diagnosis and optimal management of fetuses with congenital heart diseases. This technology enabling worldwide remote diagnosis especially underserved area not having access to facilities and represents an invaluable tool for a better understanding and interpretation of normal and abnormal fetal cardiac anatomy.

\section{References}

1. Saxena A (2018) Congenital heart disease in India: A status report. Indian Pediatr 55: 1075-1082. [crossref]

2. Ossa Galvis MM, Bhakta RT, Tarmahomed A, et al. Cyanotic Heart Disease. [Updated 2021 Jul 22]. In: StatPearls [Internet]. Treasure Island (FL): Stat Pearls Publishing; 2021 Jan.

3. Suluba E, Shuwei L, Xia Q, et al (2020) Congenital heart diseases: genetics, noninherited risk factors, and signaling pathways. Egypt J Med Hum Genet 21: 11.

4. Morris SA, Lopez KN (2021) Deep learning for detecting congenital heart disease in the fetus. Nat Med 27: 764-765. [crossref]

5. Afari HA, Davis EF, Sarma AA (2021) Echocardiography for the Pregnant Heart. Curr Treat Options Cardiovasc Med 23: 55. [crossref]

6. Wael El Guindi, Michel Dreyfus, Gabriel Carle, Nidal Alassas (2020) The Additional Diagnostic Value of the Three-Dimensional (3D) Ultrasound and Doppler angiography imaging in the prenatal diagnosis of left isomerism. Journal of Clinical Case reports and Images 2: 9-21.

7. El Guindi W, Dreyfus M, Carles G, Alassas N (2020) Can we build a House From one Brick? : Diagnosis of TGV Diagnosis from a Single Stored Heart Volume. Int J Cardiovasc Dis Diagn 28; 5: 047-053.

8. Yagel, Simcha \& Cohen, Sarah \& Shapiro, I \& Valsky, Dan (2007) 3D and 4D ultrasound in fetal cardiac scanning: A new look at the fetal heart. Ultrasound in obstetrics \& gynecology 29: 81-95. [crossref]

9. Viñals F (2011) Current experience and prospect of internet consultation in fetal cardiac ultrasound. Fetal Diagn Ther 30: 83-87. [crossref]

10. Chaoui R, Abuhamad A, Martins J, Heling K, (2020) Recent Development in Three and Four Dimension Fetal Echocardiography. Fetal Diagn Ther 47: 345-353. [crossref]

11. Karmegaraj B, Kumar S, Srimurugan B, Sudhakar A, Simpson JM, et al. (2021) 3D/4D Spatiotemporal Image Correlation (STIC) fetal echocardiography provides incremental benefit over 2D fetal echocardiography in predicting postnatal surgical approach in double-outlet right ventricle. Ultrasound Obstet Gynecol 57: 423-430. [crossref]
12. Spiridon Pepes, Fragiskos Parthenakis, Antonis Makrigiannakis, Ioannis Germanakis (2020) Electronic versus conventional Spatiotemporal Image Correlation (STIC) fetal echocardiography: a direct comparison The Journal of Maternal-Fetal \& Neonatal Medicine 15: 1-8. [crossref]

13. Hu WY, Zhou JH, Tao XY, Li SY, Wang B, et al. (2020) Novel foetal echocardiographic image processing software (5D Heart) improves the display of key diagnostic elements in foetal echocardiography. BMC Med Imaging 20: 33. [crossref]

14. Eberhard M, Sonila P (2017) "Advantages of 3D ultrasound in the assessment of fetal abnormalities:" Journal of Perinatal Medicine 45: 643-650. [crossref]

15. Chaoui R, Abuhamad A, Martins J, Heling KS, (2020) Recent Development in Three and Four Dimension Fetal Echocardiography. Fetal Diagn Ther 47: 345-353. [crossref]

16. Allan JL, Chaoui R, Copel J, DeVore G, Hecher K, et al. (2013) ISUOG Practice Guidelines (updated): sonographic screening examination of the fetal heart. Ultrasound Obstet Gynecol 41: 348-359.

17. Neel Patel, Evan Narasimhan, Anne Kennedy (2017) Fetal Cardiac US: Techniques and Normal Anatomy Correlated with Adult CT and MR Imaging. Radio Graphics 37: 1290-1303. [crossref]

18. Dursun S, Aktoz F (2020) A novel technique for determining the axis of the fetal heart: Clock position method. J Turk Ger Gynecol Assoc 21: 216-217. [crossref]

19. William E, Ruben A, Juan C, William C, Rollins RC, et al. (2009) Dextrocardia: Practical Clinical Points and Comments on Terminology. Pediatric Cardiology 31: 1-6. [crossref]

20. Panneerselvam A, Subbiahnadar P (2012) Is it dextrocardia or dextroversion? Case Reports 2012: bcr0120125493.

21. Filip Kucera (2021) Chapter 2 - Segmental approach to congenital heart disease, Editor(s): Filip Kucera, Atlas of Pediatric Echocardiography, Elsevier 27-41.

22. Oztunc F, Madazli R, Yuksel MA, Gökalp S, Oncul M (2015) Diagnosis and outcome of pregnancies with prenatally diagnosed fetal dextrocardia. J Matern Fetal Neonatal Med 28: 1104-1107. [crossref]

23. Rajesh Krishnamurthy, Johan G (2009) (Hans) Blickman, Chapter 3 - Heart, Editor(s): Johan G. Blickman, Bruce R. Parker, Patrick D. Barnes, Pediatric Radiology (Third Edition), Mosby 47-61.

24. El Guindi, Dreyfus W, Carles M, Lambert G, Herlicoviez V, et al. (2012) 3D ultrasound and Doppler angiography for evaluation of fetal cardiovascular anomalies. International Federation of Gynaecology and Obstetrics 120: 173-177. [crossref]

25. Wael El Guindi, Michel Dreyfus, Gabriel Carle, Nidal Alassas (2020) The Additional Diagnostic Value of the Three-Dimensional (3D) Ultrasound and Doppler angiography imaging in the prenatal diagnosis of left isomerism. Journal of Clinical Case reports and Images 2: 9-21.

26. El Guindi W, Dreyfus M, Carles G, Lambert V, Herlicoviez M, et al. (2012) Contribution of 3D-ultrasound in the heterotaxy syndromes: about four cases and review of the literature. G J Gynecol Obstet Biol Reprod (Paris) 41: 489-496. [crossref]

27. Carpentier A, Branchini B, Cour JC, Asfaou E, Villani M, et al. (1976) Congenital malformations of the mitral valve in children. Pathology and surgical treatment. J Thorac Cardiovasc Surg 72: 854-866. [crossref]

\section{Citation:}

El Guindi W, Youssef M, Carles G, Dreyfus M, Elsayed S. Three dimensional (3D) Ultrasound and Doppler Angiography Imaging in the Prenatal Assessment on Normal and Abnormal Fetal Cardiac Anatomy. Integr Gyn Obstet J Volume 4(3): 1-9. 